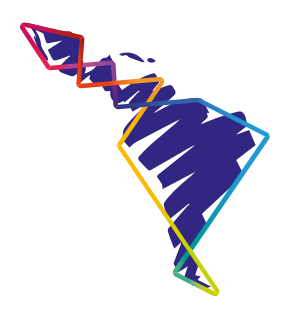

\title{
La integralidad en el abordaje de la inclusión digital
}

\section{Integrality in the approach to digital inclusion}

\section{Integralidade na abordagem da inclusão digital}

Valeria Castro-Obando ${ }^{1}$

\begin{abstract}
Resumen
El siguiente artículo presenta el concepto de la inclusión digital desde una perspectiva crítica en la que se lo plantea como un término complejo y multidimensional, que plasma un proceso que busca potenciar el uso de las tecnologías de la información y la comunicación (TIC) y desarrollar una serie de prácticas, habilidades y destrezas con el fin de que se produzca una apropiación tecnológica que dote de capacidades adecuadas y suficientes para sobrevivir en la sociedad de la información y el conocimiento (SIC). Un enfoque como este comprende tres áreas de acción distintas: el acceso a las TIC (equipamiento y desarrollo de infraestructura de telecomunicaciones), la alfabetización digital y la apropiación digital (uso de TIC y reinvención y adaptación de los usos). Una visión así de la inclusión digital requiere de la puesta en práctica de soluciones integrales que abandonen las visiones reduccionistas y unidimensionales de este problema público; adoptar estrategias que combinen acciones que incluyan la inversión y desarrollo de infraestructura de telecomunicaciones; el equipamiento y asequibilidad de las tecnologías; la calidad en los servicios, y la construcción de capacidades para la apropiación y alfabetización digital, así como considerar las necesidades diferenciadas de aquellos sectores en mayor condición de vulnerabilidad.
\end{abstract}

Palabras clave: Brecha digital, inclusión digital, tecnologías de la información y la comunicación (TIC), integralidad.

Recibido: 7-8-2021 - Aceptado: 5-10-2021

1 Investigadora. Politóloga y socióloga de la Universidad de Costa Rica (UCR), Costa Rica; Diplomada en Políticas Públicas para el Desarrollo Democrático en América Latina por la Fundación Konrad Adenauer Stiftung y la Asociación Civil de Estudios Populares de Argentina. Estudiante de la Maestría en Pensamiento Estratégico y Prospectiva de la Universidad Nacional. Investigadora del Programa Sociedad de la Información y el Conocimiento (Prosic). Correo electrónico: valeria.castro@ucr.ac.cr (D) https://orcid. org/0000-0001-7991-9308 


\begin{abstract}
The following article presents the concept of digital inclusion from a critical perspective in which it is considered as a complex and multidimensional term, which embodies a process that seeks to enhance the use of Information and Communication Technologies (ICT) and develop a series of practices, abilities and skills in order to produce a technological appropriation that provides adequate and sufficient capacities to survive in the Information and Knowledge Society (IKS). Such an approach comprises three different areas of action: access to ICTs (equipment and development of telecommunications infrastructure), digital literacy and digital appropriation (use of ICTs and reinvention and adaptation of uses). Such a vision of digital inclusion requires the implementation of integral solutions that abandon the reductionist and one-dimensional views of this public problem, adopting strategies that combine actions that include investment and development of telecommunications infrastructure, and the equipping and affordability of technologies, quality of services and the creation of capacities for appropriation and digital literacy, as well as considering the differentiated needs of those sectors in the greatest condition of vulnerability.
\end{abstract}

Keywords: digital divide, digital inclusion, information and communication technologies (ICT), integrality.

\title{
Resumo
}

O artigo a seguir apresenta o conceito de inclusão digital a partir de uma perspectiva crítica em que é considerada um termo complexo e multidimensional, que materializa um processo que busca potencializar o uso das Tecnologias da Informação e Comunicação (TIC) e desenvolver uma série de práticas, habilidades e competências para produzir uma apropriação tecnológica que proporcione capacidades adequadas e suficientes para sobreviver na Sociedade da Informação e do Conhecimento (SIC). Uma abordagem como esta compreende três diferentes áreas de ação: acesso às TIC (equipamentos e desenvolvimento de infraestrutura de telecomunicações), alfabetização digital e apropriação digital (uso das TIC e reinvenção e adaptação de usos). Tal visão de inclusão digital requer a implementação de soluções integrais que abandonem a visão reducionista e unidimensional deste problema público, adotando estratégias que combinem ações que incluem investimento e desenvolvimento de infraestrutura de telecomunicações, equipamentos e acessibilidade das telecomunicações. Tecnologias, qualidade de serviços e capacitação para apropriação e alfabetização digital, bem como considerando as necessidades diferenciadas dos setores em maior condição de vulnerabilidade.

Palavras-chave: exclusão digital, inclusão digital, tecnologias da informação e comunicação (TIC), abrangente. 


\section{Introducción}

$\mathrm{Al}$ anunciarse el estado de emergencia mundial y, posteriormente, declararse la epidemia de SARS-CoV-2 como una pandemia, los países rápidamente adoptaron todo tipo de medidas (por ejemplo, los confinamientos, el uso de mascarillas, el cierre de fronteras, el distanciamiento social en sitios públicos, el teletrabajo y la educación virtual) para frenar la expansión del virus del Covid-19. Una situación como esta implicó una disrupción en la producción, la economía y en el funcionamiento usual de las instituciones públicas. En este contexto, las tecnologías digitales fueron utilizadas como un medio para asegurar la continuidad operativa de las organizaciones y empresas, así como para mantener informada a la población en cuestiones sanitarias, evaluar necesidades y procurar que los gobiernos continuaran "brindando servicios públicos esenciales durante la crisis del Covid-19" (ONU Hábitat, Metropolis, \& CGLU, 2020, p. 2).

Estas circunstancias han conducido a una intensificación en el uso de las TIC y a impulsar el salto tecnológico en las zonas y los sectores en los que, antes de la pandemia, había una lenta acogida de las tecnologías de la información y la comunicación (TIC). Uno de los casos más evidentes es el de las plataformas digitales, las cuales, entre los meses de enero y abril del 2020, empezaron a ser empleadas con una frecuencia abrumadora. En dicho periodo la tasa de descarga de aplicaciones como Zoom, Skype y Microsoft Teams se incrementó sustancialmente a " $3.340 \%$ en América Latina y el Caribe, $2.433 \%$ en Europa y 1.509 en Estados Unidos" (Banco Interamericano de Desarrollo [BID], 2020, p. 14). Asimismo, el uso del internet a nivel global aumentó en un $70 \%$ en los primeros seis meses de la pandemia, junto con la utilización de aplicaciones móviles, las llamadas de voz y muchos otros servicios de transmisión de video (Ramos, 2020).

La digitalización acelerada de todos los sectores significó, en muchos casos, la diferencia entre la supervivencia o el cierre de negocios y la pérdida de empleos en los nichos productivos incapaces de realizar la transición hacia esquemas de trabajo digitales. La adopción de "soluciones tecnológicas y... la continuidad de los servicios de telecomunicaciones" (Comisión Económica para América Latina y el Caribe [CEPAL], 2020, p. 2) se ha visto limitada por la existencia de notorias brechas en cuanto al acceso y uso de las TIC, así como a falencias en las velocidades de conexión, las cuales impiden el acceso a servicios de banda ancha.

Por ejemplo, aunque América Latina y el Caribe (ALC) tiene un acceso a internet que está por encima del de otras regiones del mundo, este, en su mayoría, se realiza mediante dispositivos móviles, lo que restringe, de algún modo, el tipo de actividades 
que pueden realizarse. Además, en ALC, el acceso a banda ancha aún sigue siendo limitada, si se la compara con los países más desarrollados (Cepal, 2020).

Para la educación, la imposición de las cuarentenas y la imposibilidad para retomar la presencialidad obligó a los centros educativos a desarrollar estrategias para mantener la educación por medios virtuales. Es así como los países que disponían de plataformas educativas antes de la pandemia solo debieron enfocarse en la adecuación y actualización de estas herramientas, mientras que los que no contaban con ese tipo de plataformas debieron crearlas y complementarlas con clases virtuales. A pesar de esto, la educación en línea no ha sido una posibilidad para quienes no cuentan con dispositivos tecnológicos o no disponen de conexión a internet.

La crisis ocasionada por el Covid-19 ha evidenciado aún más las notables diferencias que hay con respecto al uso y apropiación de las TIC. Ello no solo limita el aprovechamiento efectivo de las tecnologías, sino que también contradice las aspiraciones que tienen las sociedades del conocimiento, en las cuales se presupone que el acceso a la información y el conocimiento debe darse en igualdad de condiciones para todas las personas y sectores, mediante el apoyo de las TIC. Conjuntamente, estas diferencias han sacado a relucir una vez más el tema de la brecha digital en tanto dicho fenómeno afecta las oportunidades de vida de millones de personas en el mundo (ONU Hábitat, Metropolis, \& CGLU, 2020) y perfilan una forma de exclusión social que eminentemente deriva de la revolución tecnológica acontecida a finales del siglo XX.

Las transformaciones estimuladas por las tecnologías asociadas a la Cuarta Revolución Industrial (como la inteligencia artificial, las redes 5G, el big data y el internet de las cosas) están provocando cambios que afectan el modo como producen las empresas, se generan los flujos de comunicación e información y se desarrollan gran parte de nuestras vidas en sociedad. Con ello, es de esperar que la digitalización de la vida humana continúe acrecentándose en las próximas décadas, convirtiéndose en la norma y no en la excepción.

El potencial efecto que esto tendrá a nivel de productividad, eficiencia y transformación de servicios marcará la diferencia entre el fracaso o el éxito de los países, las empresas, las organizaciones y las personas en el futuro; por lo cual, ante tal escenario, no se puede evitar la necesidad de generar soluciones que permitan solventar o, al menos, cerrar paulatinamente, las desigualdades que potencian la brecha digital y que amenazan con dejar a más atrás.

En ese sentido y tomando en cuenta el impacto que las tecnologías digitales tendrán en los próximos años en las diferentes áreas de la vida humana, así como las 
afectaciones generadas por la brecha digital en el uso, el acceso y apropiación de las TIC, resulta trascendental que se aborde el tema de la inclusión digital desde una perspectiva crítica que plasme la riqueza polisémica, la complejidad conceptual y las variadas aristas desde donde puede ser entendido el término.

Con este objetivo, se realizó una revisión bibliográfica de artículos de revista y documentos especializados de distintos campos del conocimiento en los que se aborda la cuestión de la inclusión digital. A partir de esto, la información recopilada fue sistematizada y analizada en cinco ejes, que ayudaron en el desarrollo de este documento. A este efecto, se presenta una conceptualización de la brecha digital como antípoda y fenómeno que justifica las intervenciones de inclusión digital, junto con las definiciones y las perspectivas principales desde donde se define qué es la inclusión digital. Seguidamente, se ahonda en las críticas que han esbozado en contra de la inclusión digital, para luego precisar en las condiciones que inciden y afectan en la misma. Una vez que han sido establecidos dichos aspectos se propone a la integralidad como el enfoque central para orientar las intervenciones de política pública, en materia de inclusión digital.

\section{La antesala del fenómeno: La brecha digital}

En términos generales, la brecha digital alude a las desigualdades que afectan el acceso a diverso tipo de tecnologías (computadoras, teléfonos móviles, red de banda ancha, infraestructura de telecomunicaciones) a distintos segmentos de la población (Levis, 2017). Esto produce una nueva forma de marginalización social en la que las personas son limitadas de los beneficios y potencialidades que ofrecen las tecnologías (Ribeiro-Rosa, 2013); así quedan relegadas de recursos básicos que requieren para poder vivir de forma digna.

Desde esta concepción, la brecha digital puede ser entendida como una restricción a las TIC y demás innovaciones tecnológicas, aun cuando estas son necesarias y forman parte del espacio vital donde deben desenvolverse (Chacón, Ordóñez \& González, 2017). Debido a que el acceso suele ser impedido por la carencia de medios financieros para poder comprar aparatos o adquirir ciertos servicios como el internet, la brecha digital suele ser considerada como una forma de exclusión social que se origina en disparidades socioeconómicas.

Sin embargo, si bien los factores económicos inciden de forma importante en la brecha digital, estos no son los únicos aspectos que intervienen. De hecho, las limitaciones también pueden ser consideradas como la conjunción de diferencias institucionales y estructurales, las cuales llevan a la débil infraestructura, carencias de conectividad, la poca asequibilidad de las TIC y a la falta de habilidades 
y conocimientos básicos para el manejo de las tecnologías (Alcalá, 2020). Es así como la brecha digital puede ser considerada como la "desigualdad entre aquellos que tienen acceso a las TIC y aquellos que no, o que teniendo acceso, están en desventaja, por infraestructura, capacitación y/o equipamiento" (Chacón, Ordóñez, \& González, 2017, p. 144).

El reconocimiento de esta multiplicidad de manifestaciones de la brecha digital ha llevado a una evolución del concepto, entendiéndolo no solo como un problema de acceso, sino como un aspecto que se expresa en el grado de alfabetización digital y en la apropiación de las tecnologías por parte de una población. Esto quiere decir que la brecha digital "es un concepto complejo, multidimensional, que conglomera una serie de desigualdades de la era digital" (Amador-Zamora, 2020, p. 224) y, por tanto, no se puede considerar que esta se comporta como una sola. En consecuencia, se puede afirmar que esta se manifiesta en dos niveles distintos, como una brecha de acceso (conectividad) y como una brecha que concierne a la capacidad y calidad de uso de las TIC (alfabetización digital). Además, al existir diversas tecnologías, la brecha digital puede ser examinada en cada tecnología en particular; lo usual es analizar en las TIC más comunes como las computadoras, los teléfonos móviles o las redes de internet, aunque también es posible determinar la brecha en tecnologías más incipientes como los drones (Amador-Zamora, 2020).

Una de las implicancias que deriva de la condición de multidimensionalidad que caracteriza la brecha digital es el hecho de que existe una amplia variedad de elementos que la potencian. Amador-Zamora (2020), siguiendo el enfoque propuesto por la Organización de las Naciones Unidas para la Educación, la Ciencia y la Cultura (Unesco), menciona un conjunto de factores socioeconómicos que inciden en la brecha digital entre los que se encuentran: los ingresos (asequibilidad), la ubicación geográfica (rural/urbano), la edad, el sexo, el idioma, el nivel educativo, el empleo y la integridad física (accesibilidad).

La preocupación por el acceso a las TIC ha llevado a los gobiernos a impulsar acciones para fortalecer el acceso a las tecnologías, al dotar de equipamiento tecnológico y redes a centros educativos e instituciones públicos, y expandir los servicios de internet fijo y móvil a los hogares. Es entonces cuando en los países se empieza a hablar de inclusión digital y de su importancia como un medio para estimular el desarrollo socioeconómico y un "derecho social que los Estados deben asegurar, dejando en un plano secundario el formidable negocio que la expansión tecnodigital representa para las empresas del sector informático y de telecomunicaciones" (Levis, 2017, p. 288). 
Pero, entonces, si la brecha digital constituye un fenómeno de amplia complejidad en el que se expresan la suma de un conjunto de factores que impiden el acceso, uso y aprovechamiento efectivo de las TIC y, por tanto, manifiesta una problema social que requiere de la intervención de los gobiernos para ser solventada, ¿qué es la inclusión digital?, ¿en qué se diferencia de la brecha digital?

\section{La inclusión digital: Un concepto en constante construcción}

Los procesos de transformación tecnológica han generado un cambio en el modo como operan las empresas, los Estados, las organizaciones e, inclusive, la forma como se tejen las relaciones humanas. En este entorno de alta digitalización, las TIC se han vuelto un factor dinamizador cuya afectación ha conducido a notables progresos en la automatización de procesos, volviéndolos más eficientes, rápidos y ágiles, provocando una modernización operativa, así como la necesidad de contar con capital humano capacitado para sobrevivir a esta nueva realidad.

En estas circunstancias son cada vez más comunes las referencias al tema la inclusión digital, acepción bajo la cual suele aludirse a un amplio espectro de cuestiones, cuya significación puede cambiar según los aspectos que se destaquen o el enfoque que se adopte. En ese sentido, el área de la inclusión digital puede ser considerada como un "territorio de disputas por sus concepciones y objetivos, y...en un plano singular, transmite poco de su complejo campo de significados" (Ribeiro-Rosa, 2013, p. 33).

Esto supone que existe una multiplicidad de formas con las cuales puede definirse la inclusión digital. En las acepciones más tradicionales, esta se liga al conjunto de acciones que buscan promover un mayor acceso a las TIC, ante las constantes innovaciones tecnológicas que suelen ocurrir en el mercado. Si bien una definición como esta brinda una idea general de lo que alude la inclusión digital, ofrece una visión limitada y no evidencia las dimensiones que contempla el concepto. En ese sentido, para contar con una perspectiva más completa de la inclusión, es necesario revisar otras definiciones y, en dicho ejercicio, es posible identificar tres puntos de vista distintos, aunque complementarios. Estos corresponden a la visión desde las políticas públicas, los derechos humanos y la relación con otros conceptos similares, tales como la alfabetización y la literacidad digital o la instrucción mediática.

En el primero de estos puntos de vista, la inclusión digital es asociada con las intervenciones públicas que se desarrollan a través de las políticas, programas y proyectos a través de los cuales se pretende equipar a una población determinada de tecnologías diversas, donde es común la dotación de equipos como celulares y computadoras, además de servicios como el internet. Dichas intervenciones 
funcionan como estrategias de inserción social sobre todo para aquellas comunidades en las que aún no han llegado dichos avances tecnológicos, lo que hace que, en esta óptica, la inclusión digital sea concebida como una respuesta institucional en la que el Estado responde a las demandas insatisfechas de la ciudadanía ante un contexto de cambio tecnológico (Chacón, Ordóñez, \& González, 2017).

Como política pública la inclusión digital puede ser concebida como aquella que puede contribuir con tres objetivos distintos, el desarrollo económico, la resolución de problemas sociales y el desarrollo multidimensional de las personas. Como elemento que fomenta el desarrollo económico, el objetivo de la inclusión digital es crear capacidades en la fuerza laboral con el fin de mejorar su inserción sociolaboral; mientras que cuando el objetivo de la es incidir en la resolución de problemas sociales, se "apuesta al poder de las tecnologías como catalizadoras de cambios" (Ribeiro-Rosa, 2013, p.37). En la tercer vertiente, la inclusión digital se centra en las personas y a partir de eso enfatiza el uso de las TIC como una medio para "desarrollarse en un escenario social que exige el uso y el conocimiento de nuevas herramientas y recursos digitales" (Ribeiro-Rosa, 2013, p.37).

La distinción entre los objetivos que puede tener la inclusión digital es importante, pues contribuye a representar, de algún modo, los distintos abordajes que los países pueden tener a la hora de responder a las demandas sociales. La selección de un enfoque $u$ otro determina elegir entre la universalización de estos bienes o servicios, y la focalización que implica que un bien o servicio sea brindado a quienes no pueden obtenerlo por sus propios medios. Asimismo, la anteposición entre la universalización y la focalización plantea un dilema con respecto a si el acceso y uso de las TIC debe ser un derecho que el Estado debe promover para toda la población o si esto constituye una responsabilidad individual.

En la perspectiva de los derechos humanos, se considera que la inclusión digital constituye una dimensión innata a la condición de la ciudadanía, en tanto resulta necesaria para estimular el desarrollo de una serie de habilidades y conocimientos que son indispensables para poder actuar autónomamente en las sociedades contemporáneas. Para las personas, esto supone la capacidad de estar integradas a los avances tecnológicos, aprovechando las ventajas que estas pueden aportar (Chacón, Ordóñez, \& González, 2017) y, por tanto, implica la igualdad de oportunidades a recursos que hoy resultan esenciales para actividades tan diversas como el recibir formación educativa (Alcalá, 2020), poder desempeñarse en un puesto de trabajo, ingresar a contenidos informativos y de entretenimiento, entre muchos otros.

De igual modo, debido a que las TIC propician la diseminación de la información y facilitan los flujos de comunicación, se considera que la inclusión digital también 
está ligada al cumplimiento de garantías individuales y sociales básicas, tales como la libertad de expresión y la democratización de los medios de comunicación (Ribeiro-Rosa, 2013), ya que las TIC contribuyen a potenciar estos derechos.

Otra de las formas en las que se vincula la inclusión digital a los derechos humanos tiene que ver con el llamado derecho al acceso a las tecnologías de la información, comunicación, conocimiento y aprendizajes digitales (TICCAD), que deriva de la Declaración Universal de Derechos Humanos y el Pacto Internacional de Derechos Económicos, Sociales y Culturales que "reconoce el derecho de las personas a participar en el progreso científico y de acceder a un fondo comunal de conocimiento difundido" (Alcalá, 2020, pp. 10-11). El TICCAD refiere a la posibilidad de acceder a conocimientos de diversas áreas del conocimiento, a prácticas educativas y a "una instrucción técnica, profesional y generalizada; con objeto del cabal e íntegro desarrollo de la personalidad y consolidación del respeto a los derechos humanos" (Alcalá, 2020, p. 10) mediante las TIC.

En contraste, las concepciones que visualizan a la inclusión digital como un concepto relacionado con otros similares, generalmente tienden a definir el término con un enfoque englobador que usualmente comprende elementos de diversas acepciones como las de alfabetización y la literacidad digital, la apropiación tecnológica y la instrucción mediática, las cuales serán explicadas a continuación con el fin de diferenciarlas entre sí e identificar qué aspectos recogen las definiciones de inclusión digital que toman como base a estos conceptos. Mientras que la alfabetización digital refiere a las habilidades básicas que son requeridas para utilizar adecuadamente las TIC, la literacidad digital alude a la adquisición de conocimientos tecnológicos y al desarrollo de capacidades suficientes para emplear lo aprendido en diferentes contextos, lo que supone hacer un "uso social de las habilidades en las TIC en su día a día, en una acción consciente frente a sus necesidades" (Ribeiro-Rosa, 2013, p. 37).

En línea con la definición anterior se encuentra la noción de apropiación tecnológica que implica que las personas dejen de ser consumidoras de las tecnologías y desarrollen una comprensión profunda de estas, lo cual les sirva para apropiarse de ellas (Chacón, Ordóñez \& González, 2017), e integrar las TIC como una parte integral de sus vidas, innovando el modo como las utilizan. Al adquirirse este tipo de mejoras se debe fomentar la instrucción mediática de las personas, entendida esta como una habilidad que permite asumir una actitud crítica con respecto a la información de los diversos medios de comunicación (Ribeiro-Rosa, 2013) y que implica no solo saber cómo usar las TIC, sino también entender los riesgos que vienen aparejados con su uso. 
A partir de lo señalado previamente, la inclusión digital puede ser entendida como una serie de prácticas, habilidades y destrezas a través de las cuales se busca potenciar el uso de las TIC, para evitar limitarse a una utilización básica y dotar de capacidades críticas que permitan una apropiación efectiva de la tecnología. Ello contempla considerar la inclusión digital como un proceso que requiere de transformaciones mentales y culturales que deben ocurrir en las personas, con el fin de que se produzca una apropiación tecnológica real, que dote de las capacidades adecuadas y suficientes para sobrevivir en la SIC y, además, potencie una comprensión crítica de la era tecnológica en la que nos encontramos. Un enfoque así de la inclusión digital determina que este concepto abarca tres áreas de acción distintas: el acceso a las TIC (equipamiento y desarrollo de infraestructura de telecomunicaciones), la alfabetización digital y la apropiación digital (uso de TIC y reinvención y adaptación de los usos).

\section{Críticas a la noción de la inclusión digital}

Aunque hoy es innegable el valor de la inclusión digital, se cuestiona el hecho de que este tipo de esfuerzos no siempre sean realizados de forma homogénea, sino que, por el contrario - en no pocas veces- han tendido a reproducir un "estándar de desigualdad, alcanzando primero a regiones de capitalismo más avanzado y clases económicas más elevadas en todo el mundo" (Ribeiro-Rosa, 2013, p. 35). Esta tendencia parece confirmarse, sobre todo si se considera que aunque el 60 $\%$ de la población mundial está en línea, la mayoría de las personas que pueden acceder a servicios de internet viven en países con mayor desarrollo (Opp, 2021), lo que evidencia una diferencia importante con respecto a quienes residen en otras regiones del mundo. Asimismo, la realidad resulta más dramática en zonas con menor desarrollo, pues en dichos países, solo 1 de cada 5 personas se encuentra en línea (Opp, 2021), lo que significa una limitación para acceder a servicios digitales de diversa índole y, a su vez, muestra que la conectividad se está convirtiendo en un impedimento creciente en la vida de las personas.

Con todo ello, crece la idea de que el acceso a dispositivos electrónicos es un aspecto que ayuda a mejorar la vida de los sectores más pobres de la sociedad; no obstante, no existen "asideros factuales que revelen beneficios concretos en cuestiones sensibles como la lucha contra la pobreza y la desigualdad o mejoras en sanidad o educación (ni datos empíricos que lo justifican" (Levis, 2017, p. 287). De hecho, el disponer de una tecnología no necesariamente lleva a una disminución de las brechas sociales y tampoco supone una transformación estructural de la desigualdad, por lo que se puede cuestionar que el desarrollo tecnológico produzca una mejora automática en el cierre de desigualdades. 
En línea con esta crítica de los discursos más optimistas de la inclusión digital destaca la posición que considera que bajo las opiniones que buscan fomentar una mayor inclusión digital se "esconde una lucha encarnizada por el control de los dispositivos de conexión y por la hegemonía sobre las normas y los sistemas técnicos" (Levis, 2017, p. 284) vinculados a una nueva actividad lucrativa. De la mano de esta intencionalidad, se cuestiona el hecho de que internet se haya convertido en un espacio que cristaliza el "objetivo principal del capitalismo contemporáneo: la creación de un espacio mundial de libre comercio sin regulaciones estatales" (Levis, 2017, p. 286).

Aunque a primera vista, estos señalamientos parecen indicar únicamente aspectos negativos que podrían inducir a un descontento con respecto al impacto que producen las acciones de inclusión digital que se realizan, no deben ser interpretadas de ese modo, sino que más bien deben ser vistas como una mirada crítica y realista que muestra el entorno en que acontecen los procesos de inclusión digital en el mundo, entre las regiones e, inclusive, a lo interno de los países. Por ello, no deben ser descartados y más bien, deben ser tomados como aspectos de mejora o claves para desarrollar estrategias de inclusión digital que trabajen sobre la base de estas falencias.

\section{¿Qué afecta la inclusión digital?}

La inclusión digital como la antípoda de la brecha digital también puede verse afectada por diversos factores entre los que pueden mencionarse la existencia de infraestructura de telecomunicaciones, el acceso a servicios TIC de calidad (Alcalá, 2020), la presencia de capital humano calificado para el uso y prestación de servicios, y las habilidades y conocimientos tecnológicos que posee la población (Comisión Económica para América Latina, [Cepal], 2020).

De los anteriores, la infraestructura de telecomunicaciones (también llamada infraestructura digital) es la cara olvidada de la inclusión digital, pues cuando se pretende realizar intervenciones para mejorar aspectos como la conectividad, se suele dar por sentado que dicho problema se resuelve únicamente con la dotación de equipo tecnológico. Sin embargo, dicha visión resulta paradójica, ya que la infraestructura constituye la base a través de la cual se construyen las condiciones físicas (por ejemplo, torres, ductos, postes, cableado estructurado, fibra óptica, antenas y radiobases, entre otras) que permiten dotar de servicios de conectividad. Por ende, ningún proceso de inclusión digital puede ser llevado a cabo si se carece de una infraestructura de telecomunicaciones adecuada; es más, acciones como el equipamiento de dispositivos (como computadoras, celulares, tabletas) no tienen sentido y pueden terminar siendo contraproducentes. 
Esto significa que para que se puedan aprovechar las potencialidades de las TIC, primero se deben establecer condiciones óptimas que generen conectividad, lo que implica priorizar el desarrollo de las infraestructuras digitales (Asociación Interamericana de Empresas de Telecomunicaciones [Asiet], 2019) como paso previo en la ejecución de cualquier acción de inclusión digital. Ello no ocurre de la noche a la mañana, sino que, por el contrario, suele suceder de manera paulatina y, además, requiere de importantes inversiones (Brichetti, Cavallo, \& Serebrisky, 2020) las cuales, generalmente, son realizadas por el sector privado (operadores de telecomunicaciones) de acuerdo con la demanda del mercado a menos que los despliegues de infraestructura sean llevados a cabo mediante fondos de acceso universal, que son recursos que los Estados disponen para fomentar la "universalización de los servicios de banda ancha"(Cepal, 2011, párr. 1) y dotar de equipamiento a ciertos sectores de la población, entre otros objetivos.

El despliegue de infraestructura de telecomunicaciones no solo contribuye a crear condiciones de conectividad para impulsar los procesos de inclusión digital, sino que también afecta la calidad de los servicios digitales que se brindan. Habilitar servicios de alta calidad supone que se alcance una conectividad en la que las redes (móviles y fijas) sean estables, se incremente la cobertura de estas y, en general, se cuente con mejor ancho de banda, lo que a su vez genera menores tiempos de latencia. Ahora bien, lo que se considera como un servicio de alta calidad puede variar según las necesidades de los sectores y organizaciones, así como entre países, regiones y personas; además conforme avanzan las tecnologías, el estándar puede evolucionar en el tiempo.

Por ejemplo, de acuerdo con la Cepal (2020), hoy se considera que las velocidades de internet inferiores a los 5,5 Mpbs, aunque ofrecen la posibilidad de conectarse a acceder a contenidos en línea, solo sirven para usos básicos, no permiten realizar dos actividades simultáneamente y pueden limitar aplicativos requeridos para acceder al teletrabajo o a clases virtuales (ver tabla 1). 


\section{Tabla 1}

Funcionalidades según velocidades de descarga de banda ancha

\begin{tabular}{l|l|l}
\hline \multicolumn{1}{c|}{ Baja } & \multicolumn{1}{c|}{ Media } & \multicolumn{1}{c}{ Alta } \\
\hline \multicolumn{1}{c}{$5,5 \mathrm{Mbps}$} & \multicolumn{1}{c}{$18,5 \mathrm{Mbps}$} & \multicolumn{1}{c}{ Más de 25 Mbps } \\
\hline $\begin{array}{l}\text { Permite utilizar funciones } \\
\text { como correo electrónico, } \\
\text { vídeo básico y transmisión } \\
\text { directa de audio y video } \\
\begin{array}{l}\text { a través de internet } \\
\text { (streaming). }\end{array}\end{array}$ & $\begin{array}{l}\text { Permite realizar } \\
\text { simultáneamente dos } \\
\text { funciones básicas y una } \\
\text { actividad en línea de alta } \\
\text { demanda. }\end{array}$ & $\begin{array}{l}\text { Permite realizar } \\
\text { simultáneamente funciones } \\
\text { básicas y funciones de alta } \\
\text { demanda. }\end{array}$ \\
$\begin{array}{l}\text { No permite el teletrabajo. } \\
\text { Permite el teletrabajo y } \\
\text { la educación en línea de } \\
\text { manera simultánea. }\end{array}$ & $\begin{array}{l}\text { Permite el teletrabajo y } \\
\text { la educación en línea de } \\
\text { manera simultánea. }\end{array}$ \\
en línea. & & \\
\hline
\end{tabular}

Nota. La tabla ejemplifica el tipo de actividades que pueden ser realizadas, según diferentes tipos de velocidades de banda ancha. Tomado de la Comisión Económica para América Latina y el Caribe, 2020.

Las velocidades expuestas en la Tabla 1 ejemplifican, dentro de la región de América Latina y Caribe (ALC), lo que hoy puede ser considerado como banda ancha; sin embargo, en otros países, como los europeos, un estándar como el de más de $25 \mathrm{Mbps}$ puede no ser lo deseable.

Con la promulgación de la Agenda Digital para Europa, la Unión Europea (UE) estableció la meta de lograr que toda la población europea contara con "cobertura de banda ancha rápida de como mínimo 30 Mbps a más tardar en 2020" (Comisión Europea, s. f., p.1), lo que evidencia que una velocidad como esta constituye el nuevo estándar mínimo a partir del cual deben mejorarse la conectividad disponible.

Otro de los aspectos que incide en los procesos de inclusión digital tiene que ver con los conocimientos y destrezas con los que cuentan las personas para poder hacer un uso adecuado de las tecnologías, a la vez que para apropiárselas. Si bien esto se relaciona con la formación de habilidades técnicas con los que se forma para el uso de las TIC (Alcalá, 2020) y el tipo de contenidos que se imparten en los centros educativos; también refiere a las habilidades digitales que posee el personal docente. En una era en la que la transformación digital aparece a la orden del día, las brechas en las capacidades TIC de una población repercuten en las oportunidades de vida futuras de las personas, y resulta muy probable que la ausencia de conocimientos restringa la posibilidad de acceder a ocupaciones de mejor remuneración y más especializadas, en las que no sea necesario el uso 
intensivo de labores manuales y más bien se requiera del manejo de TIC para llevar a cabo los procesos productivos.

Todos estos aspectos obligan a repensar la forma como se está generando la inclusión digital e implican la necesidad de asumir una perspectiva crítica frente a ello. Para esto resulta fundamental reconocer la complejidad inherente a la brecha digital, en tanto fenómeno multicausal y multidimensional que requiere de la puesta en práctica de un abordaje integral que abandone las visiones reduccionistas sobre este problema público.

\section{La integralidad en el centro de los esfuerzos de inclusión digital: Claves para mejorar las políticas existentes}

Abordar la inclusión digital desde un enfoque integral supone asumir una posición holística frente a la brecha digital, al reconocer los elementos que potencian su cierre y cuáles lo limitan. En ese sentido y a partir de lo expuesto en los apartados previos, se considera que el abordaje integral de la inclusión digital debe basarse en, al menos, cinco elementos principales:

1) Inversión y desarrollo de infraestructura de telecomunicaciones

2) Acceso, equipamiento y asequibilidad de las tecnologías

3) Calidad de tecnologías y servicios de telecomunicaciones

4) Educación, apropiación y alfabetización digital

5) Necesidades de las poblaciones vulnerables

\section{Inversión y desarrollo de infraestructura de telecomunicaciones}

Necesariamente, se requiere continuar estimulando el desarrollo y despliegue de infraestructura de telecomunicaciones, pues como se señaló en los acápites anteriores, este aspecto resulta estratégico a la hora de fomentar la digitalización de los distintos sectores productivos y garantizar el acceso a una buena conectividad (Ramos, 2020). Asimismo, considerando que las inversiones suelen ser onerosas y que estas ocurren de manera paulatina, resulta indispensable que se generen incentivos legales que, de algún modo, potencien la inversión por parte de los entes operadores de telecomunicaciones; mientras que los Estados, desde la inversión pública, deben fortalecer los mecanismos de acceso y servicio universal, con el fin de que las poblaciones más desfavorecidas puedan contar con tecnologías y servicios de conectividad.

De igual modo, la inversión debe evitar concentrarse en solo unas áreas, ya que ello produce brechas entre diversas regiones, por lo que, en la medida en que sea 
posible, se debe procurar impulsar el despliegue homogéneo de la infraestructura de telecomunicaciones. Hacerlo no solo posibilita mayor cobertura y acceso a las TIC, sino que también potencia la conectividad como un atractivo para estimular el desarrollo de los territorios.

\section{Acceso, equipamiento y asequibilidad}

Las acciones de inclusión digital también deben combinar intervenciones que garanticen que el acceso a las TIC y a los servicios de telecomunicaciones sean asequibles para la población, es decir, que estos puedan ser comprados con los recursos que las personas dispongan. Si los precios de las tecnologías y lo servicios de telecomunicaciones son muy elevados, esto se convertirá en un obstáculo para poder adquirirlos, sobre todo para quienes carezcan de los medios económicos suficientes para hacerlo.

\section{Calidad de tecnologías y servicios de telecomunicaciones}

Junto con lo anterior, es indispensable asegurar la calidad de los servicios y tecnologías que se ponen a disposición de las personas, debido a que el acceso a un bajo nivel de conectividad constituye una barrera para el uso efectivo de las TIC, pues limita las tecnologías a sus usos básicos e imposibilita el acceso a plataformas o aplicativos especiales que pueden requerirse a nivel educativo o laboral. Por lo tanto, los estándares mínimos de los servicios de telecomunicaciones deben mejorar en el tiempo, en función de los avances en la calidad de los servicios, así como adaptarse a las necesidades diferenciadas de los sectores (familias, instituciones públicas, centros educativos, empresas, organizaciones).

\section{Educación, apropiación y alfabetización digital}

Generar mayor alfabetización digital y, por ende, más apropiación tecnológica requiere del desarrollo paulatino de un conjunto de habilidades digitales que tienen que ver no solo con el uso básico de las TIC por parte de estudiantes y docentes, sino también con la creación de ofertas pedagógico-formativas que permitan la incorporación de las nuevas tecnologías a las aulas, estimulando la utilización innovadora y disruptiva de las mismas. En este contexto, concientizar y fortalecer las capacidades de la ciudadanía para hacerle frente a los peligros que pueden enfrentar al utilizar tecnologías o estar en línea (Alcalá, 2020) constituye un aspecto de atención prioritaria que se debe atender.

El incremento en el uso de dispositivos tecnológicos y aplicaciones virtuales no solo ha maximizado su presencia en nuestras sociedades, sino que también ha provocado el despliegue masivo de informaciones y datos que transitan por 
espacios virtuales y plataformas interoperables a través de las cuales se intercambian procesos en grandes cantidades de datos. En un entorno de gran dinamismo y constante digitalización, los comportamientos e información de las personas en el ciberespacio adquieren un valor comercial altamente transable (Levis, 2017) que puede ser explotado ilegalmente. Esto obliga a implementar medidas que aseguren la protección de la privacidad de las personas, eviten el robo de datos e información personal y sensible (Cepal, 2020) e, inclusive, fortalezcan las capacidades de seguridad cibernética de los Estados.

\section{Necesidades de las poblaciones vulnerables}

Igualmente, cualquier esfuerzo de inclusión digital que se pretenda desarrollar debe tomar en cuenta el modo como los aspectos geográficos, socioeconómicos, de género y etarios inciden y afectan diferenciadamente el acceso, uso y apropiación de las tecnologías. Esto es de especial importancia para las poblaciones en situación de vulnerabilidad ya que estas suelen verse afectadas por la presencia de desventajas estructurales "relacionadas a la pobreza y la exclusión educativa, laboral y social" (Meresman \& Ullmann, 2020, p. 8) que potencian aún más su vulnerabilidad.

Esto demanda la incorporación del "principio de universalidad en el acceso a los servicios sociales desde un enfoque de derechos y... ser sensibles a las diferencias y especificidades de cada grupo, buscando superar activamente las brechas y desigualdades vigentes" (Meresman \& Ullman, 2020, p. 8). Por ejemplo, con la población con discapacidad, la brecha digital evidencia "un entorno eminentemente digitalizado en el que se consolida el papel de las nuevas tecnologías como factor indiscutible de inclusión social" (Fundación Adecco, 2020, p. 5) el cual no necesariamente toma en cuenta las necesidades de esta población.

En ese sentido, para generar estrategias que efectivamente incorporen las necesidades de estos grupos, hay que identificar sus demandas y determinar cuáles deben ser atendidas primero y si se requiere de acciones focalizadas. Todo esto implica una revisión de los modos de articular e integrar las "políticas públicas y activos sociales y comunitarios que son imprescindibles para el desarrollo inclusivo" (Meresman \& Ullman, 2020, p. 9) en las estrategias de inclusión digital. Es así como las políticas para cerrar la brecha digital deben incluir acciones para incrementar la conectividad a través del acceso a dispositivos tecnológicos, sobre todo para aquellos sectores en mayor condición de vulnerabilidad y personas adultas mayores, así como el "establecimiento de partenariados con el sector privado para ampliar el acceso a la infraestructura digital" (ONU Hábitat, Metropolis, \& CGLU, 2020, p. 8). 


\section{Conclusiones}

Las tecnologías digitales forman parte de la realidad cotidiana, estas han llegado para quedarse y, muy probablemente, continúen integrándose cada día más a nuestras vidas. Por ello, es de esperar que su rol dentro de las sociedades será cada vez más preponderante, lo que plantea el desafío de gestionar el proceso de transformación digital en el que nos encontramos insertos, así como actuar, decidir y desarrollar acciones que estén encaminadas al cierre de la brecha digital.

Las personas no pueden continuar estando excluidas de los beneficios y potencialidades de esta nueva era tecnológica, además que ello, con el tiempo, solo contribuirá a fomentar y exacerbar aún más las desigualdades socio-estructurales que ya de por sí afectan a nuestras sociedades. Incluir digitalmente a las personas, a las empresas, a las organizaciones y a las regiones que están rezagadas en lo tecnológico ofrece la posibilidad de que estas puedan ser dotadas con equipamiento, acceso y conocimientos que les permitan su tránsito y la supervivencia en las transformaciones tecnológicas por acontecer en los años venideros.

Asumiendo que ese será el panorama futuro, resulta indispensable que las TIC se conviertan en un factor que potencie la inclusión social, en lugar de generar exclusión, ya que, de modo contrario, se violenta el acceso a recursos que hoy son necesarios para vivir, se refuerzan los mecanismos de marginalización social y se exacerban las vulnerabilidades enfrentadas por aquellos sectores de la población que ya antes de la pandemia estaban excluidos de los progresos tecnológicos.

En ese sentido, podemos afirmar que la inclusión digital hoy debe ser vista no solo como un problema público y una expresión de desigualdad del siglo XXI, sino también como un derecho humano que debe ser reconocido por los Estados; se debe entender que esta nueva forma de marginalización social, propugnada por las tecnologías, requiere de la puesta en práctica de soluciones integrales en las que se responda, de manera concreta y efectiva, a las demandas insatisfechas de una ciudadanía que debe hacerle frente a un contexto incierto de constante cambio tecnológico. 


\section{Referencias}

Alcalá, M. G. (2020). Retos del derecho de acceso a las tecnologías de la información y comunicación para la alfabetización y aprendizaje digital en México durante el COVID-19. Ius Comitialis, 3(6), 7-35.

Amador-Zamora, A. (2020). Acceso y uso de las TIC en los hogares costarricenses. En Programa Sociedad de la Información y el Conocimiento (Ed.), Informe hacia la sociedad de la información y el conocimiento 2020 (pp. 223-277). Prosic-UCR.

Asociación Interamericana de Empresas de Telecomunicaciones. (23 de octubre del 2019). Taller Regional LACTLD. La infraestructura digital: Un elemento estratégico para el desarrollo de los países. Asociación Interamericana de Empresas de Telecomunicaciones. https://asiet.lat/actualidad/noticias/ la-infraestructura-digital-un-elemento-estrategico-para-el-desarrollo-de-los-paises/

Banco Interamericano de Desarrollo. (2020). El futuro del trabajo en América Latina y el Caribe. ¿Cómo puede la tecnología facilitar la recuperación del empleo tras el COVID-19? BID. http://dx.doi.org/10.18235/0002646

Brichetti, J., Cavallo, E., \& Serebrisky, T. (14 de octubre del 2020). La digitalización de los servicios de infraestructura como fuente de crecimiento inclusivo. Detrás de la Escena. Banco Interamericano de Desarrollo. https://blogs.iadb.org/ideas-que-cuentan/es/la-digitalizacion-de-los-servicios-de-infraestructura-como-fuente-de-crecimiento-inclusivo/

Comisión Económica de América Latina y el Caribe. (2020). Universalizar el acceso a las tecnologías digitales para enfrentar los efectos del COVID-19. Informe N. 76 Especial Covid-19. https://repositorio.cepal.org/bitstream/handle/11362/45938/4/ S2000550_es.pdf

Comisión Económica de América Latina y el Caribe. (2011). Uso de los fondos de acceso universal de telecomunicaciones en países de América Latina y el Caribe. Comisión Económica de América Latina y el Caribe. CEPAL. https://www.cepal.org/es/publicaciones/3912-uso-fondos-acceso-universal-telecomunicaciones-paises-america-latina-caribe

Comisión Europea. (s. f.). Mercado único digital: Banda ancha y comunicaciones electrónicas. Fichas temáticas del semestre europeo. Comisión Europea. https://ec.europa.eu/info/sites/default/files/file_import/ european-semester_thematic-factsheet_digital-single-market-broadband_es.pdf

Chacón-Penagos, Á. M., Ordóñez-Córdoba, J. A., \& Anichiarico-González, A. M. (2017). Hacia el reconocimiento de la inclusión digital como un derecho fundamental en Colombia. Vniversitas, (134), 139-168. 
Levis, D. (2017). Inclusión digital no es inclusión social: De la ilusión de libertad al hiperconsumismo tecnototalitario. Psicología, Conocimiento y Sociedad, 7(2), 209- 225.

Meresman, S., \& Ullmann, H. (2020). COVID-19 y las personas con discapacidad en América Latina: Mitigar el impacto y proteger derechos para asegurar la inclusión hoy y mañana. Comisión Económica de América Latina y el Caribe. https://www.cepal.org/ es/publicaciones/46278-covid-19-personas-discapacidad-america-latina-mitigar-impacto-proteger-derechos

ONU Hábitat, Metropolis, \& Ciudades y Gobiernos Locales Unidos. (2020). Tecnologías digitales y la pandemia de COVID-19. Informe y Nota de aprendizaje. Experiencia de aprendizaje en vivo: Más allá de la respuesta inmediata al brote de COVID-19. https:// www.uclg.org/sites/default/files/eng_briefing_technology_es.pdf

Opp, R. (14 de julio del 2021). La brecha digital en evolución. Programa Naciones Unidas para el Desarrollo. https://www.undp.org/blogs/evolving-digital-divide

Ramos, M. (30 de julio del 2020). COVID-19 podría ampliar la brecha digital. Esto es lo que se necesita ahora. Foro Económico Mundial. https://www.weforum.org/ agenda/2020/07/covid-19-could-widen-the-digital-gap-here-is-what-is-needed-now/

Ribeiro Rosa, F. (2013). Inclusión digital como política pública: Disputas en el campo de los Derechos Humanos. Sur. Revista Internacional De Derechos Humanos, 10(18), 33-55. 
